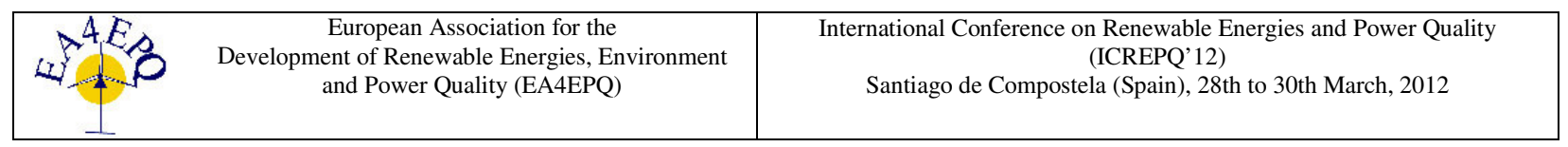

\title{
An Improved Bayesian-based Approach for Short Term Photovoltaic Power Forecasting in Smart Grids
}

\author{
A. Bracale ${ }^{1}$, P. Caramia ${ }^{1}$, U. De Martinis ${ }^{2}$, A. R. Di Fazio ${ }^{3}$ \\ ${ }^{1}$ Department for Technologies, \\ University Parthenope of Napoli,Italy \\ Centro Direzionale di Napoli, Is. C4 - 80143 Napoli (Italy) \\ Fax number:+39 081 5476777, e-mail: antonio.bracale@ uniparthenope.it,pierluigi.caramia@uniparthenope.it \\ ${ }^{2}$ Department of Electrical Engineering \\ University Federico II of Napoli, Italy \\ Via Claudio, 21 - 80125Napoli (Italy), umberto.demartinis@ unina.it \\ ${ }^{3}$ Department DAEIMI \\ University of Cassino, Italy \\ Via G. Di Biasio, 43 - 03043 Cassino(Italy), a.difazio@unicas.it
}

\begin{abstract}
Smart grid behaviour is characterized by significant uncertainties due to the time-varying nature of powers generated by random energy sources and of load demands. These uncertainties introduce several technical problems in smart grid planning and operation and new issues have to be addressed. In this context, an important role is played by probabilistic methods aimed to forecast random power productions and load demands. This paper improves a method recently proposed in the literature to perform a very short-term forecast of the active power produced by photovoltaic systems. The method, based on the Bayesian theory, is enriched by modifying the auto-regressive time-series model to take into account the dependence of the clearness index on some meteorological variables. Moreover, sets of sample data of the clearness index and of the involved meteorological variables extracted from measurements on the basis of 15 minutes is chosen to improve the forecast of the photovoltaic active power. Numerical applications are presented to give evidence of the obtained improvements in the power forecast when the cloud cover and the above sample are considered.
\end{abstract}

\section{Key words}

Distributed generation, Photovoltaic systems, Active power production, Bayesian forecasting.

\section{Introduction}

Nowadays, the distribution systems are undergoing deep changes and are evolving in the grid of the future, known as Smart Grids (SGs). SGs are sometimes defined as complex power networks using bi-directional communications among distributed energy resources, customers and a central control system. The perspective of SGs is tofacilitate large scale penetration of distributed generation exploiting renewable energy sources, such as wind and Photo Voltaic (PV) energy systems; encourage an efficient use of energy by smart loads, including electric vehicles; reduce system losses and improve power quality $[1,2,3]$.
The SG behavior is characterized by significant uncertainties due to the time-varying nature of some renewable energy sources and of load demands. Such uncertainties introduce many technical problems in SG planning and operation and new considerations must be addressed related to f. i. short circuit analysis, steadystate analysis and power quality issues.

One of the most promising renewable energy system to be integrated in a SG are PV systems, due to the expected cost reduction and the increased efficiency of both PV panels and converter [4]. While the active power of a PV system varies according to the variation of solar radiation, these energy sources cannot be dispatched in the traditional sense and forecast information on the expected PV power production is necessary for the correct management of a SG and for making decision on the energy market [5]. An accurate prediction of PV power production can be also useful to obtain economic incentives. As an example, in Italy the mean daily error on the forecasted PV active power must to be less than 10 $\%$ for 300 days in one year to obtain incentives from the Authority [6].

An accurate forecasting of the PV power production needs to consider the dependence of solar radiation on meteorological variables, such as cloud cover, ambient temperature, relative humidity [7, 8, 9]. Such dependences are very important when short-term horizons are concerned but the influence of the single meteorological variable depends on the particular site in which the PV system is installed. In addition, the inclusion of an high number of meteorological variables can significantly increase computational efforts in the application of the forecasting methods.

In the relevant literature, several methods have been proposed for PV active power forecasting, characterised by different theories and different time horizons. As an example, in [10] artificial neural networks are adopted to perform a long term forecast using as input solar irradiation, temperature and wind speed. In [11] a Markov Chain model based on operating data of the PV 
power production is applied to a short and medium term forecast. In [12] a two-stage method is used to predict hourly value of the PV power for horizon up to 36 hours. In [13] Kalman filters are applied to predict sub-hourly and hourly PV power production using solar irradiance as input.

Recently, in [14] a new Bayesian theory-based approach has been proposed to perform a very short-term forecast (from one hour to few hours ahead) of the active power produced by PV systems starting from an estimation of the hourly clearness index. In particular, by using samples of only clearness index properly extracted from the measurements until the generic hour $h$, an Auto Regressive (AR) time-series model is integrated in a Monte Carlo procedure to forecast the probability density function (pdf) of the clearness index at hour $h+m$, with $m=1,2, \ldots, N_{\text {hours }}$. This approach, providing a pdf as an output, appears very useful in solving important problems of the future SGs such as risk analysis, power quality assessment and operation of storage systems.

In this paper, the proposed approach is recalled and enriched by modifying the AR time-series model to take into account the dependence of the clearness index on some meteorological variables. In addition, a different set of sample data of the clearness index with respect to the ones used in [14] is chosen to improve the PV active power forecasting (the set of sample data is extracted from measurements collected at 15 minutes instead of hourly intervals). The same set of sample data is used for the involved meteorological variables.

The paper is organized as follows. Section 2 reports the PV power model showing the analytical relationship between the active power production and the clearness index. Section 3, after briefly recalling of the Bayesian theory-based approach, illustrates the proposed improvements to better perform the PV power forecast. Numerical applications are presented in Section 4 to give evidence of the obtained improvements in the PV power forecast when the cloud cover and a set of sample data of the clearness index and of the cloud cover extracted from measurements on the basis of 15 minutes is considered. Finally, conclusion and considerations about future works are reported in Section 5.

\section{Photovoltaic Active Power Model}

The active power produced by a PV system depends on the availability of the solar radiation. An analytical relationship between the PV power production $P_{P V_{t}}$ at hour $t$ and the corresponding hourly clearness index $k_{t}{ }^{1}$ exists when the PV system is equipped with a Maximum Power Point Tracker [15-17]:

$P_{P V_{t}}=S_{C} \eta\left(T k_{t}-T^{\prime} k_{t}^{2}\right)$

where $S_{C}$ is the array surface area, $\eta$ is the efficiency of the PV system, and $T$ and $T^{\prime}$ are defined as:

\footnotetext{
${ }^{1}$ The hourly clearness index $k_{t}$ at hour $t$ is defined as the ratio of the corresponding hourly total solar radiation on an horizontal plane $I_{t}$ to the extra-terrestrial hourly total solar radiation $I_{0}$.
}

$T=\left[\left(R_{b}+\rho \frac{1-\cos \gamma}{2}\right)+\left(\frac{1+\cos \gamma}{2}-R_{b}\right) p\right] r_{d} \frac{H_{0}}{3600}$

$T^{\prime}=\left(\frac{1+\cos \gamma}{2}-R_{b}\right) q r_{d} \frac{H_{0}}{3600}$

where: $R_{b}$ is the ratio of beam radiation on a tilted surface to that on a horizontal surface at any time, $\rho$ is the reflectance of the ground, $\gamma$ is the inclination of the array surface to the horizontal plane, $r_{d}$ is the ratio between diffuse radiation in hours and diffuse radiation in a day, $H_{o}$ is the extra-terrestrial total solar radiation, and $p, q$ are coefficients reported in [16], which link the diffuse fraction of the hourly total solar radiation on horizontal plane with the hourly clearness index.

The analysis of the relationship (1) clearly reveals that $P_{P V_{t}}$ depends only on $k_{t}$ once the hour of the day, the installation site and the technical characteristics of the PV system are assigned. The hourly clearness index $k_{t}$ at hour $t$ is a random variable modelling the random behaviour of the solar energy source; then, $P_{P V_{t}}$ is a random variable too. It follows that, once the pdf of $k_{t}$ is known, the pdf of $P_{P V_{t}}$ can be easily obtained using (1) and applying the fundamental theorem for function of a random variable [18].

\section{Clearness index Bayesian Forecasting}

In general, Bayesian inference is a recursive prediction algorithm that uses a priori distributions of prior random parameters and measurements to update a posteriori distributions for the next time increment. The a posteriori distributions describe uncertainty about a priori distributions given by a set of sample data measured previous the forecast [19].

In [14], a new Bayesian theory-based approach is proposed to perform a very short-term forecast of the active power produced by PV systems starting from an estimation of the hourly clearness index. In particular, it forecasts at hour $h$ the pdf of the clearness index $k_{t}$ at hour $t=h+m$. Without loss of generality, and for the sake of simplicity, the following analysis is referred to the case of $m=1$.

The starting points of the Bayesian forecasting approach are the assumptions regarding:

i. the choice of the analytical expression of the pdf modeling the random variable to be forecasted;

ii. the definition of the time series model representing the linkage between the statistical figures (i.e., the mean value) of the random variable to forecast and its measured values before the time of forecast;

iii. the selection of the measurements from which to extract vector data needed to calculate an approximation (samples) of the a posteriori distributions of the prior random parameters.

In [14] the hourly clearness index $k_{h+1}$ at hour $h+1$ is modeled with a conditional pdf $f_{k_{h+1}}\left(k_{h+1} \mid C_{h+1}, \lambda_{h+1}\right)$ described by a modified Gamma distribution, whose parameters are $C_{h+1}$ and $\lambda_{h+1}$ : 
$f_{k_{h+1}}\left(k_{h+1} \mid C_{h+1}, \lambda_{h+1}\right)=C_{h+1} \frac{\bar{k}_{u}-k_{h+1}}{\bar{k}_{u}} e^{\lambda_{h+1} k_{h+1}}$

where $\bar{k}_{u}$ is the upper bound of the observed values of $k_{t}$ and the parameters $C_{h+1}$ and $\lambda_{h+1}$ are defined as:

$C_{h+1}=\frac{\lambda_{h+1}^{2} \bar{k}_{u}}{\left(e^{\lambda_{h+1} \bar{k}_{u}}-1-\lambda_{h+1} \bar{k}_{u}\right)}$

$\lambda_{h+1}=\frac{\left(2 F_{h+1}-17.519 e^{-1.3118 F_{h+1}}-1062 e^{-5.0426 F_{h+1}}\right)}{\bar{k}_{u}}$

with:

$F_{h+1}=\frac{\bar{k}_{u}}{\bar{k}_{u}-\mu_{k_{h+1}}}$

where $\mu_{k_{h+1}}$ is the mean value of the hourly clearness index $k_{t}$ at hour $h+1$. Assuming that the upper bound $\bar{k}_{u}$ is known, the distribution parameters $C_{h+1}$ and $\lambda_{h+1}$ only depend on the mean value $\mu_{k_{h+1}}$. Consequently, the only parameter of the pdf in (2) is the mean value $\mu_{k_{h+1}}$ at hour $h+1$.

The time series model adopted in [14] is a first-order AR model linking the mean value $\mu_{k_{h+1}}$ at hour $h+1$ and the value $k_{h}$ of the hourly clearness index measured at hour $h$ and described by the following relashionship:

$\mu_{k_{h+1}}=\alpha_{1} k_{h}+\alpha_{0}$

where $\alpha_{0}$ and $\alpha_{1}$ are the AR model parameters, assumed as prior random parameters of the Bayesian inference.

Finally, in [14] the vector data $\boldsymbol{k}_{\boldsymbol{t}}$ of the hourly clearness index used to evaluate the samples of the a posteriori pdfs of $\alpha_{0}$ and $\alpha_{1}$ is extracted from measurements collected at hourly intervals in the site in which the PV system is installed.

In this paper some assumptions adopted in [14] are modified in order to improve the prediction and reduce the error on forecasting the PV active power. In particular, with reference to the second assumption, a new AR timeseries model, able to take into account meteorological variables strongly correlated to the clearness index, is proposed. Concerning the third assumption, sets of sample data of the clearness index and of involved meteorological variables, extracted from measurements collected at 15 minutes intervals, is used. In the following, the proposed improvements are described in details.

\section{A. AR time-series model with meteorological variables}

The AR time-series model described in (3) presents the advantage of requiring only measurements of clearness index. However, it has been shown in the relevant literature [7-9] that a most accurate forecasting can be performed taking into account the dependence of the clearness index on some meteorological variables, such as cloud cover, ambient temperature and humidity. Then, in this paper the following AR time-series model is adopted:

$\mu_{k_{h+1}}=\alpha_{1} k_{h}+\beta_{1} v_{1, h}+\cdots+\beta_{M} v_{M, h}+\alpha_{0}$

where the set $\left(v_{1, h}, \ldots, v_{M, h}\right)$ is composed of $M$ meteorological variables measured at hour $h$ and the set $\left(\beta_{1}, \ldots, \beta_{M}\right)$ is composed of the corresponding AR model parameters, assumed as additional prior random parameters of the Bayesian inference.
An "off-line" correlation analysis between historical data of hourly clearness index and of other meteorological variables, measured on the site in which the PV system is installed, can help to identify what are the variables that have to be considered in (4). Obviously, such variables have to present the higher correlation value with the hourly clearness index. On the other hand, the inclusion of an high number $M$ of meteorological variables can significantly increase computational efforts in the application of the Bayesian approach. Then, the number of meteorological variables to be considered has to be chosen finding a compromise between result accuracy and computational efforts.

\section{B. Set of sample data containing measurements collected at 15 minutes intervals}

In [14] the samples of the a posteriori pdfs of $\alpha_{0}$ and $\alpha_{1}$ are evaluated by applying the Metropolis-Hasting alghoritm with sets of sample data $\boldsymbol{k}_{\boldsymbol{t}}$ extracted from measurements collected at hourly intervals until the hour $h$. Once the pdfs of $\alpha_{0}$ and $\alpha_{1}$ samples are known, they are used in (3) together with the value $k_{h}$ to obtain the samples of the pdf of $\mu_{k_{h+1}}$ at hour $h+1$. Finally, these samples are used to generate the pdf of the clearness index $f_{k_{h+1}}\left(k_{h+1} \mid \mu_{k_{h+1}}\right)$ at hour $h+1$.

In this paper, the a posteriori pdfs of $\alpha_{0}$ and $\alpha_{1}$ together with the additional a posteriori pdfs of $\beta_{1}, \ldots, \beta_{M}$ are calculated using a set of data of the clearness index and of selected meteorological variables, containing measurements collected at 15 minutes instead hourly intervals. Once the a posteriori pdfs samples are known, they are used in (4) together with the values of the clearness index and the selected meteorological variables measured in the last 15 minutes of the hour $h$ to forecast the pdf of the clearness index in the first 15 minutes of the hour $h+1^{2}$.

Starting from the knoweledge of the pdf of the clearness index forecasted at the first 15 minutes of hour $h+1$, the pdf at hour $h+1$ can be obtained in two different ways:

1. the pdf forecasted at $h+1$ is assumed to be equal to the pdf forecasted in the first 15 minutes of $h+1$;

2. the pdf forecasted at $h+1$ is assumed to be equal to the average of four pdfs forecasted at next 15 minutes intervals. While the pdf in the first 15 minutes of $h+1$ is known, the evaluation of the pdf in the next 15 minutes needs a measurement of the clearness index in the first 15 minutes of hour $h+1$ to use the AR model in (4). Being not available, an estimation of this value can be derived from the pdf of the clearness index at the first 15 minutes of $h+1$, using f. i. its mean value. Then, the last two pdfs can be achieved in a similar way.

\section{Numerical applications}

The very short term forecast of the hourly PV active power is performed with reference to a $50-\mathrm{kW}_{\mathrm{p}} \mathrm{PV}$

\footnotetext{
${ }^{2}$ Using measurements collected at 15 minutes intervals implies that the terms $k_{h}$ and $\left(v_{1, h}, \ldots, v_{M, h}\right)$ in equation (4) refer to the last 15 minutes of the hour $h$.
} 
system, characterized by $S_{C}=600 \mathrm{~m}^{2}$ and $\eta=0.09$. Solar radiation data and meteorological measurements are obtained from the website of the National Renewable Energy Laboratory ${ }^{3}$.

A correlation analysis between the clearness index and selected meteorological variables (air temperature, relative humidity, wind speed and total cloud cover - defined as the ratio in $\%$ of the sky hidden by all visible clouds), is performed by using data from January 2009 to December 2011. Figure 1 reports the time evolution of the correlation in the observed period. In order to avoid excessive computational efforts, only the meteorological variable furnishing the highest value of correlation coefficient with the hourly clearness index, i.e. the total cloud cover, is taken into account. Consequently, the AR time-series model in (4) used in the following analysis reduces to:

$\mu_{k_{h+1}}=\alpha_{1} k_{h}+\beta_{1} v_{1, h}+\alpha_{0}$

where $v_{1, h}$ is the total cloud cover measured at hour $h$ and $\beta_{1}$ is the corresponding additional prior random parameter. The forecast of the hourly PV active power is performed referring to different months in winter and summer 2009 and 2010. In the following, for the sake of brevity, the results relative to January, February, July and August 2009 are reported.

Four different cases are considered, characterized by a different choices of the AR time-series model and the measurements time interval:

a) $\mathrm{AR}$ time-series model described in (3) and measurements of the clearness index collected at hourly intervals: classical Bayesian Model (BM);

b) $\mathrm{AR}$ time-series model described in (3) and measurements of the clearness index collected at 15 minutes intervals (BMq);

c) $\mathrm{AR}$ time-series model described in (5) and measurements of the clearness index and of the total cloud cover collected at 15 minutes intervals. The pdf of the clearness index at hour $h+1$ is forecasted by adopting the first approach of Section 3.B (BMCq1);

d) as in (c) but the pdf at hour $h+1$ is forecasted by adopting the second approach of Section 3.B (BMCq2).

In all cases, the pdf of the PV active power is evaluated applying equation (1) and the mean values of the pdfs are initially used as forecasted power production at hour $h+$ 1.

First of all, the performance of the applied approaches are quantified comparing the hourly actual PV power production values $\left(P_{p v, h}\right)$ with the forecasted ones $\left(P_{p v, h}^{*}\right)$ using the mean relative errors defined as follows:

$$
\begin{aligned}
& e_{r}=\frac{1}{N} \sum_{h=1}^{N} e_{h r}=\frac{1}{N} \sum_{h=1}^{N} \frac{\left|P_{p v, h}-P_{p v, h}^{*}\right|}{P_{p v, h}} 100 \\
& e_{r n}=\frac{1}{N} \sum_{h=1}^{N} e_{h r n}=\frac{1}{N} \sum_{h=1}^{N} \frac{\left|P_{p v, h}-P_{p v, h}^{*}\right|}{P_{n}} 100
\end{aligned}
$$

\footnotetext{
${ }^{3}$ The measurements of the solar radiation belong to thetime interval [8:00, 18:00] and are collected in Colorado ( Latitude: $39.742^{\circ}$ North and Longitude: $\left.105.18^{\circ} \mathrm{West}\right)$.
}

where $N$ is the number of hours with solar radiation in the considered study period and $P_{n}$ is the rated power of the PV system $\left(P_{n}=50 \mathrm{kWp}\right)$.

The error $e_{r}$ is the most widely used in the relevant literature. The error $e_{r n}$ appears useful in practical since it weighs the absolute error of produced powers with the rated power of PV system.

Tables I show the mean relative errors (6) and (7) with reference to both a winter period (January and February 2009, Table I a) and a summer period (July and August 2009, Table I b).

The performance of the applied approaches are also quantified applying the following daily mean relative errors:

$e_{d r}=\frac{1}{H} \sum_{h=1}^{H} \frac{\left|P_{p v, h}-P_{p v, h}^{*}\right|}{P_{p v, h}} 100$

$e_{d r n}=\frac{1}{H} \sum_{h=1}^{H} \frac{\left|P_{p v, h}-P_{p v, h}^{*}\right|}{P_{p v, h}} 100$,

where $H$ is the number of hours with solar radiation in a day. In particular, Tables II show the number of days (in $\%$ of total considered days) with error $e_{d r}$ lower than $10 \%$ and $20 \%$. Table II a) refers to the winter period (January and February 2009) while Table II b) refers to the summer period (July and August 2009).

From the analysis of Tables I and II, it clearly appears that BMCq1 method guarantees the best results.

For the sake of brevity the relative errors $e_{d r n}$ are not shown. It should be noted that once again the BMCq1 method guarantees the best results in both seasons. In particular, the number of days with $e_{d r n}<5 \%\left(e_{d r n}<\right.$ $10 \%)$ is greater than $37 \%(80 \%)$ of the number of all considered days.

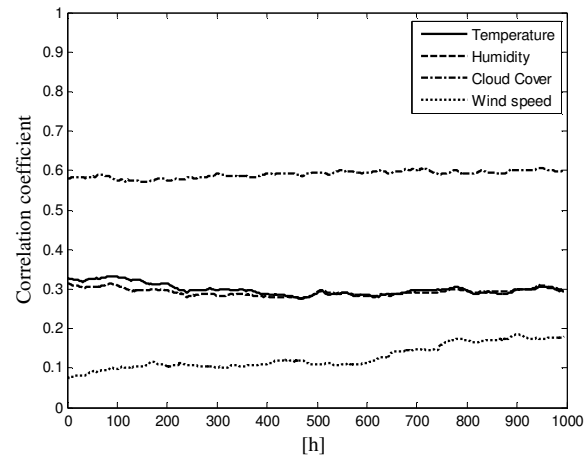

Fig. 1. Time evolution of the correlation coefficient between the clearness index and the selected meteorological variables.

Table I. - Mean errors in: (a) January and February 2009; (b)

\begin{tabular}{|c|c|c|}
\hline \multicolumn{3}{|c|}{ (a) } \\
\hline Method & $e_{r}(\%)$ & $e_{r n}(\%)$ \\
\hline BM & 41.45 & 10.26 \\
\hline $\mathrm{BMq}$ & 18.97 & 9.57 \\
\hline $\mathrm{BMCq} 1$ & 12.98 & 6.95 \\
\hline BMCq2 & 14.80 & 8.66 \\
\hline \multicolumn{3}{|c|}{ (b) } \\
\hline Method & $e_{r}(\%)$ & $e_{r n}(\%)$ \\
\hline $\mathrm{BM}$ & 33.86 & 9.84 \\
\hline $\mathrm{BMq}$ & 16.01 & 8.47 \\
\hline BMCq1 & 12.25 & 6.29 \\
\hline $\mathrm{BMCq} 2$ & 13.89 & 7.33 \\
\hline
\end{tabular}
(b) July and August 2009 
Table II. -Number of days with $e_{d r}$ lower than $10 \%$ and $20 \%$ in: (a) January and February 2009, (b) July and August 2009

(a)

\begin{tabular}{|l|c|c|}
\hline Method & $\begin{array}{c}\% \text { days with } \\
e_{d r}<10 \%\end{array}$ & $\begin{array}{c}\% \text { days with } \\
e_{d r}<20 \%\end{array}$ \\
\hline BM & 8.42 & 31.25 \\
\hline BMq & 14.58 & 65.20 \\
\hline BMCq1 & 41.66 & 83,75 \\
\hline BMCq2 & 24.33 & 72.55 \\
\hline
\end{tabular}

(b)

\begin{tabular}{|l|c|c|}
\hline Method & $\begin{array}{c}\text { \% days with } \\
e_{d r}<10 \%\end{array}$ & $\begin{array}{c}\text { \% days with } \\
e_{d r}<20 \%\end{array}$ \\
\hline $\mathrm{BM}$ & 16.07 & 50.00 \\
\hline $\mathrm{BMq}$ & 25.00 & 69.28 \\
\hline $\mathrm{BMCq} 1$ & 42.85 & 82.85 \\
\hline $\mathrm{BMCq} 2$ & 32.14 & 76.42 \\
\hline
\end{tabular}

As a further example of the obtainable results, Figs. 2 show the actual PV powers and the forecasted values versus hours obtained applying $\mathrm{BMq}$ and $\mathrm{BMCq} 1$. Fig. 2a refers to a winter day characterized by a daily error lower than $10 \%$ while Fig 2.b refers to a summer day characterized by daily error lower than 20\%. In Figs. 3 (Figs. 4) the hourly relative errors $e_{h r}$ and $e_{h r n}$ defined in (6) and (7) and calculated for the same days of Figs. 2 are plotted.

Figs. 2 clearly reveals that BMCq1 method is more able than BMq to follow fast changes of powers. Figs. 3 shows great relative error $e_{h r}$ of both methods in the hours of low $\mathrm{PV}$ produced power (and then at the beginning and the end of the "solar day"). The normalized relative error $e_{h r n}$ (Figs. 4) assumes, instead, more stable error values, since it weighs the absolute errors of produced powers with the rated value of PV system.

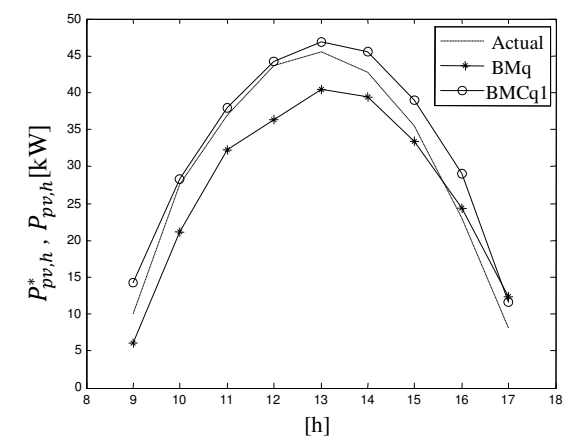

(a)

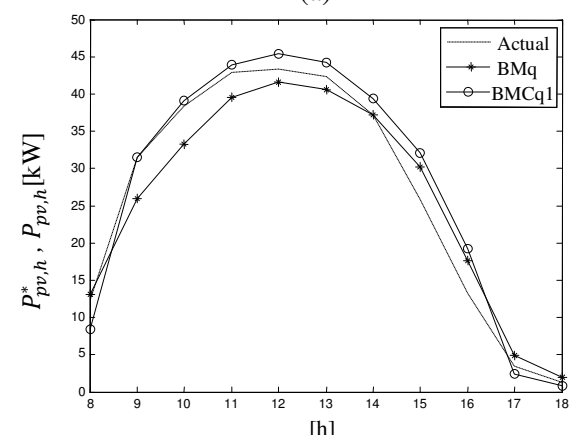

(b)

Fig. 2. Actual values, $\mathrm{BMq}$ and $\mathrm{BMCq} 1$ forecasted mean values of PV system active power versus hours: (a) winter day, (b) summer day
Finally, Fig. 5 shows the actual PV powers together with the 5th and 95th percentile values of PV hourly forecasted pdf obtained applying BMCq1 in a day of summer season with daily error greater than $20 \%$.

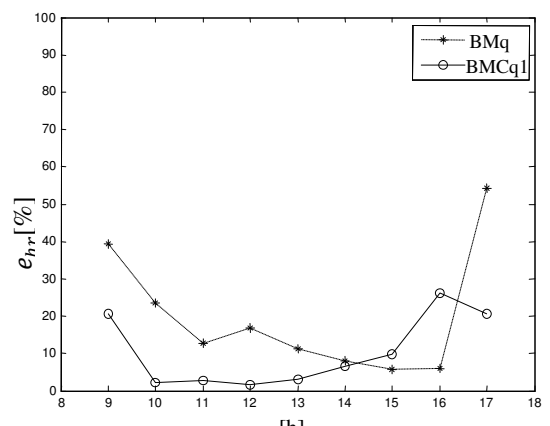

(a)

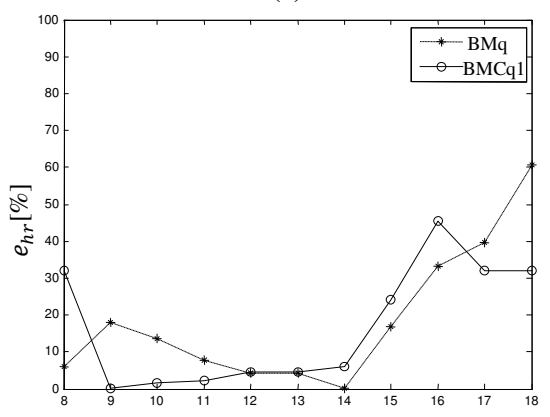

[h]

(b)

Fig. 3. BMq and BMCq1 errors $e_{h r}$ versus hours: (a) winter day, (b) summer day

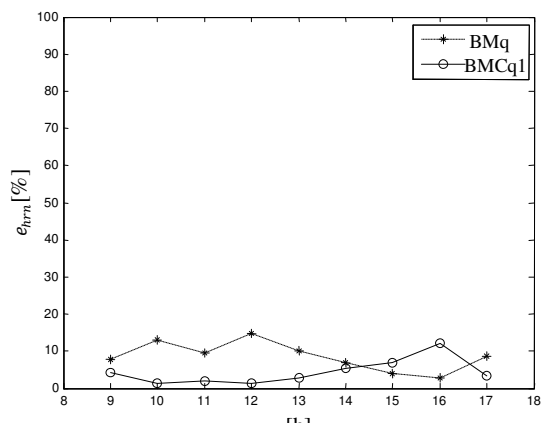

(a)

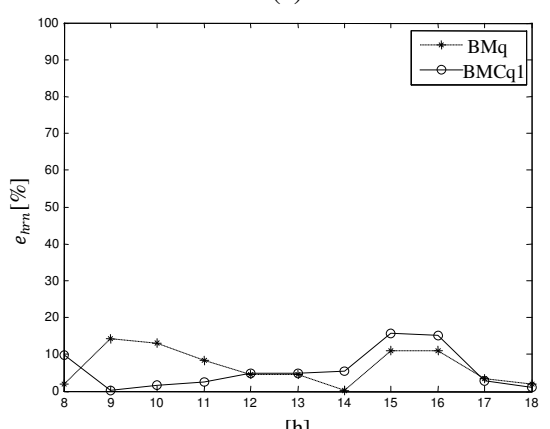

(b)

Fig. 4. BMq and BMCq1 errors $e_{h r n}$ versus hours: (a) winter day, (b) summer day 


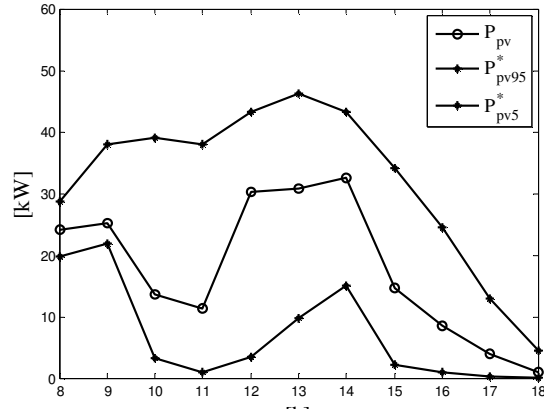

[h]

Fig. 5. PV system active power versus hours: actual values and $\mathrm{BMCq} 1$ forecasted $5^{\text {th }}$ and $95^{\text {th }}$ percentile values in a summer day.

From the analysis of Fig. 5 it is evident that, also in presence of a significant daily mean error, the measurements of the hourly active power PV production are always between the $5^{\text {th }}$ and $95^{\text {th }}$ percentile values. A similar behavior characterizes in almost all considered days.

\section{Conclusions}

A method based on the Bayesian theory has recently been proposed in literature to perform a very short term forecast of the active power produced by PV system starting from an estimation of the hourly clearness index.

In this paper, this method has been recalled and enriched in order to improve the PV power forecast. First of all, a new AR time-series model has been proposed, taking into account the dependence of some meteorological variables on the clearness index. Furthermore, measurements collected at 15 minutes instead of hourly intervals have been used to extract the set of sample data of the random variables involved in the forecasting. Numerical results have been presented.

Considering the dependence of the clearness index on the cloud cover and reducing the measurement time interval have given significant improvements in the PV power forecast. However, the forecasting errors should still be reduced, especially during the hours around sunrise and sunset; however, the proposed approach guarantees that the actual hourly PV produced powers are, in almost all considered days, between the $5^{\text {th }}$ and $95^{\text {th }}$ percentile values of forecasted power production.

With the aim of further improve the power forecast, future research will focus on the use of a multimodal pdf modelling of the clearness index and of improved AR time series models.

\section{References}

[1] C. W. Gellings, M. Samotyi, B. Howe: "The Future's Smart Delivery System", IEEE Power and Energy Magazine, Vol. 2, No. 5, pp. 40-48, September-October 2004.

[2] H. Farhangi: "The Path of the Smart Grid", IEEE Power and Energy Magazine, Vol. 8, No. 1, pp. 18-28, JanuaryFebruary 2010
[3] E. Santacana, G. Rackliffe, XiaomingFeng: "Getting Smart", IEEE Power and Energy Magazine, Vol. 8, No. 2, pp. 41-48, March-April 2010

[4] M. Liserre, T. Sauter, J. Y. Hung:"Future Energy Systems: Integrating Renewable Energy Sources into the Smart Power Grid Through Industrial Electronics", IEEE Industrial Electronics Magazine, Vol. 4, No. 1, pp. 18-37, March 2010

[5] C. V. Potter, A. Archambault, K. Westrick: "Building a smarter smart grid through better renewable energy information" (Power Systems Conference and Exposition, 2009, pages 1-5)

[6] Decreto - GU n. 197/2010: "Incentivazione della produzione di energia elettrica mediante conversione fotovoltaica della fonte solare" - Ministero dello sviluppo economico, 2010.

[7] Y. Huang, J. Lu, C. Liu, X. Xu, W. Wang, X. Zhou: "Comparative study of power forecasting methods for PV stations" (International Conferenec on Power System Technology, 2010, pages 1-6)

[8] A. Mellit, A. MassiPavan: "A 24-h forecast of solar irradiance using artificial neuralnetwork: Application for performance prediction of a grid-connectedPV plant at Trieste, Italy", Solar Energy, Vol. 84, No. 5, May 2010, pp. $807-821$

[9] D. F. Al Riza, S. I. Gilani, M. S. Aris: "Hourly Solar Radiation Estimation Using Ambient Temperature and Relative Humidity Data", International Journal of Environmental Science and Development, Vol. 2, No. 3, June 2011

[10] T. Hiyama, K. Kitabayashi: "Neural Network Based Estimation of Maximum Power Generation from PV Module Using Environmental Information" (IEEE Transactions on Energy Conversion, Vol. 12, No. 3, 1997, pages 241-247)

[11] Ying-zi Li J. Niu: “ Forecast of Power Generation for Grid-Connected Photovoltaic System Based on Markov Chain" (Asia Pacific Power and Energy Engineering Conference, 2009, pages 1-4)

[12] P. Bacher, H. Madsen, H. A. Nielsen: "Online Short-Term Solar Power Forecasting", Solar Energy, Volume 83, Issue 10, October 2009, pp. 1772-1783

[13] Hassanzadeh, M.; Etezadi-Amoli, M.; Fadali, M.S: "Practical approach for sub-hourly and hourly prediction of PV power output" (IEEE Conferences North American Power Symposium, 2010, pages 1-5)

[14] Bracale, P. Caramia M. Fantauzzi, A. R. Di Fazio: “ A Bayesian-based Approach for Photovoltaic Power Forecast in Smart Grids, Cigrè Symposium, Bologna, 2011.

[15] J. A. Duffie, W. A. Beckman: "Solar Engineering of Thermal Processes", Wiley Interscience, New York, Second ed., 1991

[16] J.F. Orgill, K.T.G. Hollands: "Correlation equation for hourly diffuse radiation on a horizontal surface", Solar Energy, Vol. 19 (4), 1977, pp. 357-359

[17] B. Kroposki, K. Emery, D. Myers L. Mrig: "A comparison of photovoltaic module performance evaluation methodologies for energy ratings", Proc. Of 1th WPEC, Haway, USA, 1994

[18] A. Papoulis, "Probability, random variables and stochastic processes ", McGraw-Hill NY, 1991

[19] A. Gelman, J. B. Carlin, H. S. Stern, D. B. Rubin: "Bayesian data analysis" London (UK), Chaoman\& Hall, 1995. 